\title{
A SHARP IMPROVEMENT OF FIXED POINT RESULTS FOR QUASI-CONTRACTIONS IN $b$-METRIC SPACES
}

\author{
NGUYEN VAN DUNG \\ Received 30 September, 2019
}

\begin{abstract}
In this paper, a general fixed point theorem for quasi-contractions in $b$-metric spaces, which is a sharp improvement of Amini-Harandi's result, Mitrovic and Hussain's result, and is a generalization of many $b$-metric fixed point theorems in the literature, is proved. The technique overcomes some limits in $b$-metric fixed point theory compared to metric fixed point theory. The obtained results are also supported by examples.
\end{abstract}

2010 Mathematics Subject Classification: 47H10; 54H25; 54D99; 54E99

Keywords: quasi-contraction, $b$-metric, fixed point

\section{INTRODUCTION AND PRELIMINARIES}

There have been many types of contraction conditions in metric spaces and generalized metric spaces [12], [19]. One of the most interesting types is the quasicontraction [7]. Quasi-contractions have been studied and many nice results have been proved. In [4], Bessenyei studied nonlinear quasicontractions in complete metric spaces. In [5], Bessenyei studied weak $\varphi$-quasi-contractions and presented an elementary proof for known fixed point results in the literature. In [2], Amini-Harandi proved a fixed point theorem for quasi-contraction maps in $b$-metric spaces. Recently, Mitrović and Hussain [17] established fixed point results for weak $\varphi$-quasicontractions involving comparison function in $b$-metric spaces.

Recall that the $b$-metric space is a generalization of a metric space. One of the main differences between a $b$-metric space and a metric space is that the modulus of concavity $\kappa \geq 1$ in the generalized triangle inequality, see Definition 1. (3) below. It implies that a $b$-metric is not necessarily continuous, see [3, Example 3.10] for example. It also implies that the contraction constants in certain $b$-metric fixed point theorems are in [0; $\left.\frac{1}{\kappa}\right)$ instead of [0;1), see [9, Remark 2.7] and [17, Corollary 3.5] for example. So, in many $b$-metric fixed point theorems, certain additional assumptions have been added to overcome the above difference such as the Fatou property in [2],

The work is supported by the project SPD2018.01.27 of Dong Thap University, Vietnam. 
the bounded orbit in [5] and [17]. For some recent improvements in $b$-metric fixed point theory, the reader may refer to [9], [18], [10], [14], [16], [15], [21].

In this paper, we are interested to improve the main results of [2] and [17]. By using a technical calculation that $\lambda^{n} \in\left[0 ; \frac{1}{\kappa}\right)$ for all $\lambda \in[0 ; 1)$ and all $n$ large enough, we prove a fixed point theorem for quasi-contractions in $b$-metric spaces which is a sharp improvement of the main results in [2] and [17], and is a generalization of many $b$-metric fixed point theorems in the literature. We also construct examples to support the obtained results.

Now we recall notions and results which will be useful in the next.

Definition 1 ([8], page 263). Let $X$ be a nonempty set, $\kappa \geq 1$ and $D: X \times X \rightarrow$ $[0 ; \infty)$ be a function such that for all $x, y, z \in X$,

(1) $D(x, y)=0$ if and only if $x=y$.

(2) $D(x, y)=D(y, x)$.

(3) $D(x, z) \leq \kappa[D(x, y)+D(y, z)]$.

Then

(1) $D$ is called a $b$-metric on $X$ and $(X, D, \kappa)$ is called a $b$-metric space. Without loss of generality we may assume that $\kappa$ is the smallest possible value, and it is called the modulus of concavity of the given $b$-metric.

(2) The sequence $\left\{x_{n}\right\}$ is called convergent to $x$ if $\lim _{n \rightarrow \infty} D\left(x_{n}, x\right)=0$, written by $\lim _{n \rightarrow \infty} x_{n}=x$.

(3) The sequence $\left\{x_{n}\right\}$ is called Cauchy if $\lim _{n, m \rightarrow \infty} D\left(x_{n}, x_{m}\right)=0$.

(4) The $b$-metric space $(X, D, \kappa)$ is called compete if every Cauchy sequence is a convergent sequence.

Definition 2 ([12], Definition 12.7). Let $X$ be a nonempty set, $\kappa \geq 1$ and $D$ : $X \times X \rightarrow[0, \infty)$ be a function such that for all $x, y, z \in X$,

(1) $D(x, y)=0$ if and only if $x=y$.

(2) $D(x, y)=D(y, x)$.

(3) $D(x, z) \leq D(x, y)+\kappa D(y, z)$.

Then $D$ is called a strong $b$-metric on $X$ and $(X, D, \kappa)$ is called a strong b-metric space.

Note that every strong $b$-metric is continuous, and the convergence and completeness in strong $b$-metric spaces are defined as in $b$-metric spaces.

Definition 3 ([2], Definition 2.4). A $b$-metric space $(X, D, \kappa)$ is called to have Fatou property if for all $x, y \in X$ and $\lim _{n \rightarrow \infty} x_{n}=x$ we have

$$
D(x, y) \leq \liminf _{n \rightarrow \infty} D\left(x_{n}, y\right) .
$$


Theorem 1 ([2], Theorem 2.8). Let $(X, D, \kappa)$ be a complete b-metric space having Fatou property and $f: X \rightarrow X$ be a map such that for some $\lambda \in\left[0 ; \frac{1}{\kappa}\right)$ and all $x, y \in X$,

$$
\begin{aligned}
& D(f(x), f(y)) \\
\leq & \lambda \max \{D(x, y), D(x, f(x)), D(y, f(y)), D(x, f(y)), D(y, f(x))\} .
\end{aligned}
$$

Then $f$ has a unique fixed point $x^{*}$ and $\lim _{n \rightarrow \infty} f^{n}(x)=x^{*}$ for all $x \in X$.

Theorem 2 ([5], Theorem on page 289). Assume that

(1) $(X, D, \kappa)$ is a complete metric space and $f: X \rightarrow X$ is a map such that for all $x, y \in X$,

$$
D(f(x), f(y)) \leq \varphi(\operatorname{diam} O(x, y))
$$

where $\varphi:[0 ; \infty) \rightarrow[0 ; \infty)$ is an increasing, upper semicontinuous function, $\varphi(0)=0$ and $\varphi(t)<t$ for all $t>0$, and

$$
O(x, y)=\left\{f^{n}(x), f^{n}(y): n \in \mathbb{N} \cup\{0\}\right\} .
$$

(2) Each orbit of $f$ is bounded.

Then $f$ has unique fixed point $x^{*}$ and $\lim _{n \rightarrow \infty} f^{n}(x)=x^{*}$ for all $x \in X$.

Theorem 3 ([17], Theorem 3.3). Assume that

(1) $(X, D, \kappa)$ is a complete $b$-metric space and $f: X \rightarrow X$ is a map such that for some $\lambda \geq 0$ and all $x, y \in X$,

$$
D(f(x), f(y)) \leq \lambda \operatorname{diam} O(x, y) .
$$

(2) $\lambda \in[0 ; 1)$ and each orbit of $f$ is bounded.

Then we have

(1) There exists $x^{*} \in X$ such that $\lim _{n \rightarrow \infty} f^{n}(x)=x^{*}$ for all $x \in X$.

(2) $f$ has unique fixed point $x^{*}$ if one of the following holds

(a) $f$ is continuous at $x^{*}$.

(b) D is continuous.

Theorem 4 ([17], Corollary 3.5). Let $(X, D, \kappa)$ be a complete b-metric space and $f: X \rightarrow X$ be a map such that for some $\lambda \in\left[0 ; \frac{1}{\kappa}\right)$ and all $x, y \in X$,

$$
\begin{aligned}
& D(f(x), f(y)) \\
\leq & \lambda \max \left\{D(x, y), D(x, f(x)), D(y, f(y)), \frac{D(x, f(y))}{2 \kappa}, \frac{D(y, f(x))}{2 \kappa}\right\} .
\end{aligned}
$$

Then $f$ has a unique fixed point.

Theorem 5 ([17], Corollary 3.6). Let $(X, D, \kappa)$ be a complete strong b-metric space and $f: X \rightarrow X$ be a map such that for some $\lambda \in[0 ; 1)$ and all $x, y \in X$,

$$
D(f(x), f(y)) \leq \lambda \max \{D(x, y), D(x, f(x)), D(y, f(y)), D(x, f(y)), D(y, f(x))\} .
$$

Then $f$ has a unique fixed point. 


\section{THE MAIN RESULT}

The main result is Theorem 6 below. Note that

(1) Theorem 6 is an improvement of Theorem 1 in the sense that the assumption of Fatou property is omitted, and the contraction constant $\lambda \in[0 ; 1)$.

(2) Theorem 6 is an improvement of Theorem 4 in the sense that the right side of (2.1) is greater than that of (1.4), and the contraction constant $\lambda \in[0 ; 1)$.

(3) Theorem 6 is an improvement of Theorem 5 in the sense that the strong $b$-metric is replaced by a continuous $b$-metric.

(4) Theorem 6 is a generalization of many $b$-metric fixed point theorems in the literature such as [1, Theorem 2.1], [1, Theorem 3.1], [11, Corollary 3.12], [20, Corollary 2.6].

(5) Recently, an analogue of Reich contraction in $b$-metric spaces was proved [13, Theorem 3.1]. In the proof on [13, page 85], the author claimed $\lim _{n \rightarrow \infty} d\left(x_{n+1}, T x^{*}\right)=d\left(x^{*}, T x^{*}\right)$ provided that $\lim _{n \rightarrow \infty} x_{n}=x^{*}$. Unfortunately, this claim does not hold since the $b$-metric $d$ is not necessarily continuous. In fact, the conclusion in [13, Theorem 3.1] does not hold which was proved in [9, Remark 2.7].

Theorem 6. Assume that

(1) $(X, D, \kappa)$ is a complete $b$-metric space and $f: X \rightarrow X$ is a map such that for some $\lambda \geq 0$ and all $x, y \in X$,

$$
\begin{aligned}
& D(f(x), f(y)) \\
\leq & \lambda \max \{D(x, y), D(x, f(x)), D(y, f(y)), D(x, f(y)), D(y, f(x))\} .
\end{aligned}
$$

(2) One of the following holds

(a) $D$ is continuous and $\lambda \in[0 ; 1)$.

(b) $\lambda \in\left[0 ; \frac{1}{\mathrm{~K}}\right)$.

Then $f$ has a unique fixed point $x^{*}$ and $\lim _{n \rightarrow \infty} f^{n}(x)=x^{*}$ for all $x \in X$.

Proof. For $m \leq i \leq n-1$ and $m \leq j \leq n$, from (2.1) we find that

$$
\begin{aligned}
& D\left(f^{i}(x), f^{j}(x)\right) \\
= & D\left(f f^{i-1}(x), f f^{j-1}(x)\right) \\
\leq & \lambda \max \left\{D\left(f^{i-1}(x), f^{j-1}(x)\right), D\left(f^{i-1}(x), f f^{i-1}(x)\right), D\left(f^{j-1}(x), f f^{j-1}(x)\right),\right. \\
& \left.D\left(f^{i-1}(x), f f^{j-1}(x)\right), D\left(f^{j-1}(x), f f^{i-1}(x)\right)\right\} \\
= & \lambda \max \left\{D\left(f^{i-1}(x), f^{j-1}(x)\right), D\left(f^{i-1}(x), f^{i}(x)\right), D\left(f^{j-1}(x), f^{j}(x)\right),\right. \\
& \left.D\left(f^{i-1}(x), f^{j}(x)\right), D\left(f^{j-1}(x), f^{i}(x)\right)\right\} .
\end{aligned}
$$

From (2.2), we get

$$
\max \left\{D\left(f^{i}(x), f^{j}(x)\right): m \leq i, j \leq n\right\}
$$




$$
\begin{aligned}
& \leq \lambda \max \left\{D\left(f^{i}(x), f^{j}(x)\right): m-1 \leq i, j \leq n\right\} \\
& \leq \ldots \\
& \leq \lambda^{m} \max \left\{D\left(f^{i}(x), f^{j}(x)\right): 0 \leq i, j \leq n\right\} .
\end{aligned}
$$

It implies that

$$
\max \left\{D\left(f^{i}(x), f^{j}(x)\right): 1 \leq i, j \leq n\right\} \leq \lambda \max \left\{D\left(f^{i}(x), f^{j}(x)\right): 0 \leq i, j \leq n\right\} .
$$

Since $0 \leq \lambda<1$, we see that

$$
\max \left\{D\left(f^{i}(x), f^{j}(x)\right): 0 \leq i, j \leq n\right\}=\max \left\{D\left(x, f^{i}(x)\right): 1 \leq i \leq n\right\} .
$$

So there exists $1 \leq k_{n}(x) \leq n$ such that

$$
D\left(x, f^{k_{n}(x)}(x)\right)=\max \left\{D\left(f^{i}(x), f^{j}(x)\right): 0 \leq i, j \leq n\right\} .
$$

Since $\lambda \in[0 ; 1)$, there exists $n_{0}$ such that $\lambda^{n_{0}}<\frac{1}{\kappa}$. If $k_{n}(x) \leq n_{0}$, then

$$
D\left(x, f^{k_{n}(x)}(x)\right) \leq \max \left\{D\left(x, f^{i}(x)\right): 0 \leq i \leq n_{0}\right\} .
$$

If $k_{n}(x)>n_{0}$, then using (2.3) we find that

$$
\begin{aligned}
& D\left(x, f^{k_{n}(x)}(x)\right) \\
\leq & \kappa\left[D\left(x, f^{n_{0}}(x)\right)+D\left(f^{n_{0}}(x), f^{k_{n}(x)}(x)\right)\right] \\
\leq & \kappa\left[D\left(x, f^{n_{0}}(x)\right)+\lambda^{n_{0}} \max \left\{D\left(f^{i}(x), f^{j}(x)\right): 0 \leq i, j \leq k_{n}(x)\right\}\right] \\
\leq & \kappa\left[D\left(x, f^{n_{0}}(x)\right)+\lambda^{n_{0}} \max \left\{D\left(f^{i}(x), f^{j}(x)\right): 0 \leq i, j \leq n\right\}\right] \\
= & \kappa\left[D\left(x, f^{n_{0}}(x)\right)+\lambda^{n_{0}} D\left(x, f^{k_{n}(x)}(x)\right)\right] .
\end{aligned}
$$

Note that $\lambda^{n_{0}}<\frac{1}{\kappa}$. So we get

$$
D\left(x, f^{k_{n}(x)}(x)\right) \leq \frac{\kappa}{1-\kappa \lambda^{n_{0}}} D\left(x, f^{n_{0}}(x)\right) .
$$

It follows from (2.4) and (2.5) that

$$
\max \left\{D\left(f^{i}(x), f^{j}(x)\right): 0 \leq i, j \leq n\right\} \leq \frac{\kappa}{1-\kappa \lambda^{n_{0}}} \max \left\{D\left(x, f^{i}(x)\right): 0 \leq i \leq n_{0}\right\}
$$

for all $n$. So

$$
\sup \left\{D\left(f^{i}(x), f^{j}(x)\right): 0 \leq i, j \leq \infty\right\} \leq M<\infty
$$

where

$$
M=\frac{\kappa}{1-\kappa \lambda^{n_{0}}} \max \left\{D\left(x, f^{i}(x)\right): 0 \leq i \leq n_{0}\right\} .
$$

By (2.3) we have

$$
\begin{gathered}
\sup \left\{D\left(f^{i}(x), f^{j}(x)\right): m \leq i, j \leq \infty\right\} \leq \lambda \sup \left\{D\left(f^{i}(x), f^{j}(x)\right): m-1 \leq i, j \leq \infty\right\} \\
\leq \ldots \leq \lambda^{m} \sup \left\{D\left(f^{i}(x), f^{j}(x)\right): 0 \leq i, j \leq \infty\right\} \leq \lambda^{m} M .
\end{gathered}
$$

Then

$$
\lim _{m \rightarrow \infty} \sup \left\{D\left(f^{i}(x), f^{j}(x)\right): m \leq i, j \leq \infty\right\}=0 .
$$


Therefore the sequence $\left\{f^{n}(x)\right\}$ is a Cauchy sequence. Since $X$ is complete, there exists $x^{*} \in X$ such that

$$
\lim _{n \rightarrow \infty} f^{n}(x)=x^{*}
$$

By (2.1) we get

$$
\begin{aligned}
& D\left(f^{n+1}(x), f\left(x^{*}\right)\right) \\
= & D\left(f f^{n}(x), f\left(x^{*}\right)\right) \\
\leq & \lambda \max \left\{D\left(f^{n}(x), x^{*}\right), D\left(f^{n}(x), f^{n+1}(x)\right), D\left(x^{*}, f\left(x^{*}\right)\right),\right. \\
& \left.D\left(f^{n}(x), f\left(x^{*}\right)\right), D\left(x^{*}, f^{n+1}(x)\right)\right\} .
\end{aligned}
$$

We consider two following cases.

Case 1. $D$ is continuous and $\lambda \in[0 ; 1)$.

Using (2.7) and the continuity of $D$, we obtain

$$
\begin{aligned}
D\left(x^{*}, f\left(x^{*}\right)\right) & \leq \lambda \max \left\{0,0, D\left(x^{*}, f\left(x^{*}\right)\right), D\left(x^{*}, f\left(x^{*}\right)\right), 0\right\} \\
& =\lambda D\left(x^{*}, f\left(x^{*}\right)\right) .
\end{aligned}
$$

Since $\lambda \in[0 ; 1)$, we have $D\left(x^{*}, f\left(x^{*}\right)\right)=0$. So $x^{*}$ is a fixed point of $f$.

Case 2. $\lambda \in\left[0 ; \frac{1}{\kappa}\right)$.

It follows from (2.7) that

$$
\begin{aligned}
& \liminf _{n \rightarrow \infty} D\left(f^{n+1}(x), f\left(x^{*}\right)\right) \\
\leq & \lambda \max \left\{0,0, D\left(x^{*}, f\left(x^{*}\right)\right), \liminf _{n \rightarrow \infty} D\left(f^{n}(x), f\left(x^{*}\right)\right), 0\right\} \\
= & \lambda \max \left\{D\left(x^{*}, f\left(x^{*}\right)\right), \liminf _{n \rightarrow \infty} D\left(f^{n}(x), f\left(x^{*}\right)\right)\right\} .
\end{aligned}
$$

By (2.8), we consider two following subcases.

Subcase 2.1. $\liminf _{n \rightarrow \infty} D\left(f^{n+1}(x), f\left(x^{*}\right)\right) \leq \lambda D\left(x^{*}, f\left(x^{*}\right)\right)$

We find that

$$
D\left(x^{*}, f\left(x^{*}\right)\right) \leq \kappa\left[D\left(x^{*}, f^{n+1}(x)\right)+D\left(f^{n+1}(x), f\left(x^{*}\right)\right)\right] .
$$

From (2.6) and (2.9) we deduce that

$$
\liminf _{n \rightarrow \infty} D\left(f^{n+1}(x), f\left(x^{*}\right)\right) \geq \frac{1}{\kappa} D\left(x^{*}, f\left(x^{*}\right)\right) .
$$

On the contrary, suppose that $x^{*} \neq f\left(x^{*}\right)$. Note that $0 \leq \lambda<\frac{1}{\kappa}$. Then

$$
\liminf _{n \rightarrow \infty} D\left(f^{n+1}(x), f\left(x^{*}\right)\right) \leq \lambda D\left(x^{*}, f\left(x^{*}\right)\right)<\frac{1}{\kappa} D\left(x^{*}, f\left(x^{*}\right)\right) .
$$

This is a contradiction with (2.10). Therefore $x^{*}=f\left(x^{*}\right)$.

Subcase 2.2. $\liminf _{n \rightarrow \infty} D\left(f^{n+1}(x), f\left(x^{*}\right)\right) \leq \lambda \liminf _{n \rightarrow \infty} D\left(f^{n}(x), f\left(x^{*}\right)\right)$. 
From $\liminf _{n \rightarrow \infty} D\left(f^{n}(x), f\left(x^{*}\right)\right)=\liminf _{n \rightarrow \infty} D\left(f^{n+1}(x), f\left(x^{*}\right)\right)$ and $0 \leq \lambda<\frac{1}{\kappa}$ we have $\liminf _{n \rightarrow \infty} D\left(f^{n}(x), f\left(x^{*}\right)\right)=0$. So there exists a subsequence $\left\{f^{k_{n}}(x)\right\}$ of $\left\{f^{n}(x)\right\}$ such that

$$
\lim _{n \rightarrow \infty} f^{k_{n}}(x)=f\left(x^{*}\right)
$$

Note that

$$
D\left(x^{*}, f\left(x^{*}\right)\right) \leq \kappa\left[D\left(x^{*}, f^{k_{n}}(x)\right)+D\left(f^{k_{n}}(x), f\left(x^{*}\right)\right)\right] .
$$

Letting $n \rightarrow \infty$ in (2.12) and using (2.11), (2.6) we obtain $D\left(x^{*}, f\left(x^{*}\right)\right)=0$. Then $x^{*}=f\left(x^{*}\right)$.

By the conclusions of Case 1 and Case 2, we find that $f$ has a fixed point $x^{*}$ and by (2.6), $\lim _{n \rightarrow \infty} f^{n}(x)=x^{*}$.

Finally, we prove the uniqueness of the fixed point of $f$. Indeed, let $x^{*}, y^{*}$ be two fixed points of $f$. From (2.1) we have

$$
\begin{aligned}
& D\left(x^{*}, y^{*}\right) \\
= & D\left(f\left(x^{*}\right), f\left(y^{*}\right)\right) \\
\leq & \lambda \max \left\{D\left(x^{*}, y^{*}\right), D\left(x^{*}, f\left(x^{*}\right)\right), D\left(y^{*}, f\left(y^{*}\right)\right), D\left(x^{*}, f\left(y^{*}\right)\right), D\left(y^{*}, f\left(x^{*}\right)\right)\right\} \\
= & \lambda D\left(x^{*}, y^{*}\right) .
\end{aligned}
$$

Since $\lambda \in[0 ; 1)$, we obtain $D\left(x^{*}, y^{*}\right)=0$, that is, $x^{*}=y^{*}$. Then the fixed point of $f$ is unique.

Next we present some examples to illustrate the obtained result. The following example shows there exists the map $f: X \rightarrow X$ so that Theorem 6 is applicable but Theorem 1, Theorem 4 and Theorem 5 are not.

Example 1. Let $X=\mathbb{R}$, and $D(x, y)=|x-y|^{2}$ for all $x, y \in X$, and the map $f: X \rightarrow$ $X$ be defined by $f(x)=\frac{3}{4} x$ for all $x \in X$. Then

(1) $(X, D, \kappa)$ is a complete $b$-metric space with the modulus of concavity $\kappa=2, D$ is continuous, and the condition (2.1) holds for all $\lambda \in\left[\frac{3}{4}, 1\right)$. Then Theorem 6 is applicable to $f$.

(2) The conditions (1.1) and (1.4) do not hold for all $\lambda \in\left[0, \frac{1}{\kappa}\right)$. Then Theorem 1 and Theorem 4 are not applicable to $f$.

(3) $D$ is not a strong $b$-metric. Then Theorem 5 is not applicable to $f$.

Proof. (1). It is easy to check that $(X, D, \kappa)$ is a complete $b$-metric space with the modulus of concavity $\kappa=2, D$ is continuous, and the condition (2.1) holds for all $\lambda \in\left[\frac{3}{4}, 1\right)$. Then Theorem 6 is applicable to $f$.

(2). For $x=0, y=1$ and $\lambda \in\left[0, \frac{1}{\mathrm{~K}}\right)=\left[0, \frac{1}{2}\right)$, we find that

$$
\begin{aligned}
& D(f(0), f(1)) \\
= & \frac{9}{16}
\end{aligned}
$$




$$
\begin{aligned}
& \geq \lambda \\
& =\lambda \max \{D(0,1), D(0, f(0)), D(1, f(1)), D(0, f(1)), D(1, f(0))\} .
\end{aligned}
$$

This proves that conditions (1.1) and (1.4) do not hold for all $\lambda \in\left[0, \frac{1}{\kappa}\right)$. Then Theorem 1 and Theorem 4 are not applicable to $f$.

(3). On the contrary, suppose that $D$ is a strong $b$-metric. Then there exists $K \geq 1$ such that for all $x, y, z \in X$,

$$
|x-y|^{2} \leq|x-z|^{2}+K|z-y|^{2} .
$$

For $n \in \mathbb{N}$, and $x_{0}=\frac{1}{n}, y_{0}=1+\frac{1}{n}, z_{0}=1$ we have

$$
\begin{gathered}
\left|x_{0}-y_{0}\right|^{2}=1 \\
\left|x_{0}-z_{0}\right|^{2}+K\left|z_{0}-y_{0}\right|^{2}=\left(\frac{1}{n}-1\right)^{2}+\frac{K}{n^{2}}=1+\frac{1-2 n+K}{n^{2}} .
\end{gathered}
$$

So for $n>K$ we have

$$
\left|x_{0}-z_{0}\right|^{2}+K\left|z_{0}-y_{0}\right|^{2}<1=\left|x_{0}-y_{0}\right|^{2} \text {. }
$$

It is a contradiction to (2.13). Then $D$ is not a strong $b$-metric, and Theorem 5 is not applicable to $f$.

The following example shows that the continuity of $D$ in Theorem 6. (2a) and the condition $\lambda \in\left[0 ; \frac{1}{\kappa}\right)$ in Theorem 6. (2b) are essential.

Example 2. Let $X=\left\{0,1, \frac{1}{2}, \ldots, \frac{1}{n}, \ldots\right\}$, and

$$
D(x, y)= \begin{cases}0 & \text { if } x=y \\ 1 & \text { if } x \neq y \in\{0,1\} \\ |x-y| & \text { if } x \neq y \in\{0\} \cup\left\{\frac{1}{2 n}: n=1,2, \ldots\right\} \\ \frac{1}{4} & \text { otherwise, }\end{cases}
$$

and let $f: X \rightarrow X$ be defined by

$$
f(x)= \begin{cases}1 & \text { if } x=0 \\ \frac{1}{10 n} & \text { if } x=\frac{1}{n}, n=1,2, \ldots\end{cases}
$$

Then

(1) $(X, D, \kappa)$ is a complete $b$-metric space with the modulus of concavity $\kappa=4$.

(2) There exist $\lambda \geq 0$ such that the contraction condition (2.1) holds for all $x, y \in$ $X$.

(3) $D$ is not continuous and $\lambda \in\left[\frac{1}{\kappa} ; 1\right)$.

(4) $f$ is fixed point free.

Proof. By [9, Example 2.6], $(X, D, \kappa)$ is a complete metric-type space with the modulus of concavity $\mathrm{\kappa}=4$. Then $(X, D, \kappa)$ is also a complete $b$-metric on $X$ with the modulus of concavity $\kappa=4$. The remaining conclusions were proved in $[9, \mathrm{Ex}-$ ample 2.6] and [9, Remark 2.7]. 
The following example shows that the assumption of bounded orbit in Theorem 2 and Theorem 3 is essential. Moreover, for the case of unbounded orbit, the value $\operatorname{diam} O(x, y)$ cannot be replaced by $\max \{d(x, f(y)), d(y, f(x))\}$. However, the value $\operatorname{diam} O(x, y)$ can be replaced by $d(x, y)$ in the class of complete regular semimetric spaces, which is a generalization of the class of complete $b$-metric spaces, see [6, Theorem 1].

Example 3. Let $X=\{1,2,3, \ldots\}, d(x, y)=|x-y|$ for all $x, y \in X, f(x)=x+2$ for all $x \in X$ and $\varphi:[0 ; \infty) \rightarrow[0 ; \infty)$ be defined by

$$
\varphi(t)= \begin{cases}\frac{2}{3} t & \text { if } t \in[0 ; 3) \\ t-1 & \text { if } t \geq 3\end{cases}
$$

Then we have

(1) $(X, d)$ is a complete metric space, and $\varphi$ is an increasing, upper semicontinuous function, $\varphi(0)=0$ and $\varphi(t)<t$ for all $t>0$. In particular, $(X, d)$ is also a complete $b$-metric space.

(2) Every orbit of $f$ is unbounded. So $\operatorname{diam} O(x, y)=\infty$ and then (1.3) holds for all $x, y \in X$ and all $\lambda \in(0 ; 1)$.

(3) $d(f(x), f(y)) \leq \varphi(\max \{d(x, f(y)), d(y, f(x))\})$ for all $x, y \in X$. Then (1.2) holds for all $x, y \in X$.

(4) $f$ is fixed point free.

Proof. (1). It is clear that $(X, d)$ is a complete metric space, and $\varphi$ is an increasing, upper semicontinuous function, $\varphi(0)=0$ and $\varphi(t)<t$ for all $t>0$.

(2). For all $x \in X$ we have $O(x)=\{x, x+2, x+4, \ldots\}$ which is an unbounded orbit of $f$.

(3). Let $x, y \in X$. We may assume that $x<y$. The we have

$$
d(f(x), f(y))=|x-y|=y-x
$$

and

$$
d(x, f(y))=y-x+2 \geq 3 .
$$

Then we have

$$
\varphi(\max \{d(x, f(y)), d(y, f(x))\})=\varphi(d(x, f(y)))=y-x+1>d(f(x), f(y)) .
$$

This proves that (1.2) holds for all $x, y \in X$.

(4). Since $f(x)=x+2$ for all $x \in X, f$ is fixed point free.

\section{ACKNOWLEDGEMENTS}

The author sincerely thanks the anonymous reviewers for their helpful comments during the preparation of the paper. 


\section{REFERENCES}

[1] S. Aleksic, H. Huang, Z. D. Mitrovic, and S. Radenovic, "Remarks on some fixed point results in b-metric spaces," J. Fixed Point Theory Appl., vol. 20, no. 4, pp. 1-17, 2018, doi: 10.1007/s11784018-0626-2.

[2] A. Amini-Harandi, "Fixed point theory for quasi-contraction maps in $b$-metric spaces," Fixed Point Theory, vol. 15, no. 2, pp. 351-358, 2014.

[3] T. V. An, L. Q. Tuyen, and N. V. Dung, "Stone-type theorem on $b$-metric spaces and applications," Topology Appl., vol. 185-186, pp. 50-64, 2015, doi: 10.1016/j.topol.2015.02.005.

[4] M. Bessenyei, "Nonlinear quasicontractions in complete metric spaces," Expo. Math., vol. 33, no. 4, pp. 517-525, 2015, doi: 10.1016/j.exmath.2015.03.001.

[5] M. Bessenyei, “The contraction principle in extended context," Publ. Math. Debrecen, vol. 89, pp. 287-295, 2016, doi: 10.5486/pmd.2016.7657.

[6] M. Bessenyei and Z. Páles, "A contraction principle in semimetric spaces," J. Nonlinear Convex Anal., vol. 18, no. 3, pp. 515-524, 2017.

[7] L. B. Ćirić, “A generalization of Banach's contraction principle," Proc. Amer. Math. Soc., vol. 45, pp. 267-273, 1974, doi: 10.1090/s0002-9939-1974-0356011-2.

[8] S. Czerwik, "Nonlinear set-valued contraction mappings in b-metric spaces," Atti Sem. Math. Fis. Univ. Modena, vol. 46, pp. 263-276, 1998.

[9] N. V. Dung and V. T. L. Hang, "On relaxations of contraction constants and Caristi's theorem in b-metric spaces," J. Fixed Point Theory Appl., vol. 18, no. 2, pp. 267-284, 2016, doi: 10.1007/s11784-015-0273-9.

[10] N. Hussain, Z. D. Mitrović, and S. Radenović, "A common fixed point theorem of Fisher in $b$ metric spaces," Rev. R. Acad. Cienc. Exactas Fís. Nat. Ser. A Mat. RACSAM, vol. 113, no. 2, pp. 949-956, 2019, doi: 10.1007/s13398-018-0524-x.

[11] M. Jovanović, Z. Kadelburg, and S. Radenović, "Common fixed point results in metric-type spaces," Fixed Point Theory Appl., vol. 2010, pp. 1-15, 2010, doi: 10.1155/2010/978121.

[12] W. Kirk and N. Shahzad, Fixed point theory in distance spaces. Cham: Springer, 2014. doi: 10.1007/978-3-319-10927-5.

[13] X. Lv and Y. Feng, "Some fixed point theorems for Reich type contraction in generalized metric spaces," J. Math. Anal., vol. 9, no. 5, pp. 80-88, 2018.

[14] R. Miculescu and A. Mihail, "New fixed point theorems for set-valued contractions in $b$-metric spaces," J. Fixed Point Theory Appl., vol. 19, no. 3, pp. 2153-2163, 2017, doi: 10.1007/s11784016-0400-2.

[15] Z. D. Mitrović and S. Radenović, "A common fixed point theorem of Jungck in rectangular $b$ metric spaces," Acta Math. Hungar., vol. 153, no. 2, pp. 401-407, 2017, doi: 10.1007/s10474017-0750-2.

[16] Z. D. Mitrović, "A note on a Banach's fixed point theorem in $b$-rectangular metric space and b-metric space," Math. Slovaca, vol. 68, no. 5, pp. 1113-1116, 2018, doi: 10.1515/ms-2017-0172.

[17] Z. D. Mitrovic and N. Hussain, "On weak quasicontractions in b-metric spaces," Publ. Math. Debrecen, vol. 94, pp. 29-38, 2019, doi: 10.5486/pmd.2019.8260.

[18] A. Petruşel and G. Petruşel, "A study of a general system of operator equations in $b$-metric spaces via the vector approach in fixed point theory," J. Fixed Point Theory Appl., vol. 19, no. 3, pp. 1793-1814, 2017, doi: 10.1007/s11784-016-0332-x.

[19] B. E. Rhoades, "A comparison of various definition of contractive mappings," Trans. Amer. Math Soc., vol. 226, pp. 257-290, 1977, doi: 10.1090/S0002-9947-1977-0433430-4.

[20] J. R. Roshan, N. Shobkolaei, S. Sedghi, and M. Abbas, "Common fixed point of four maps in b-metric spaces," Hacet. J. Math. Stat., vol. 43, no. 4, pp. 613-624, 2014. 
[21] T. Suzuki, "Fixed point theorems for single-and set-valued F-contractions in $b$-metric spaces," $J$. Fixed Point Theory Appl., vol. 20, no. 1, pp. 1-12, 2018, doi: 10.1007/s11784-018-0519-4.

Author's address

Nguyen Van Dung

Faculty of Mathematics Teacher Education, Dong Thap University, Cao Lanh City, Dong Thap Province, Vietnam

E-mail address: nvdungedthu.edu.vn 